\title{
Laser Annealing of Low Temperature Deposited Silicon Waveguides
}

\author{
Y. Franz ${ }^{1}$, A. F. J. Runge ${ }^{1}$, S. Z. Oo ${ }^{1,2}$, N. Healy ${ }^{1}$, G. Martinez-Jimenez ${ }^{1}$, A. Z. Khokhar ${ }^{1,2}$, A. Tarazona ${ }^{2}$, \\ H. M. H. Chong ${ }^{2}$, S. Mailis ${ }^{1}$, and A. C. Peacock ${ }^{1}$ \\ 1. Optoelectronics Research Centre, University of Southampton, Highfield, Southampton, SO17 1BJ, U. K. \\ 2. School of Electronics and Computer Science, University of Southampton, Highfield, Southampton, SO17 1BJ, U. K. \\ y.franz@soton.ac.uk
}

\begin{abstract}
We report the fabrication of low temperature deposited polysilicon waveguides using a laser annealing process. Micro-Raman and XRD measurements reveal the quasi-single crystal-like quality of the material, which exhibits low optical losses of $5.13 \mathrm{~dB} / \mathrm{cm}$.

OCIS codes: (160.6000) Semiconductor materials; (230.0250) Optoelectronics; (140.3390) Laser materials processing.
\end{abstract}

\section{Introduction}

Silicon ( $\mathrm{Si}$ ) is an excellent material for integrated photonics devices as its high refractive index allows for small device footprints, with the potential for integration with electronics. To date, crystalline silicon (c-Si) remains the most widely used form of this material, owing to its exceptional optical properties. However, c-Si photonic devices are relatively difficult to integrate with the electronic layers due to fabrication constraints. Thus more recently there has been increased interest in deposited silicon materials, including hydrogenated amorphous silicon (a-Si:H) [1], silicon nitride $(\mathrm{SiN})$ and polycrystalline silicon (poly-Si) [2]. Although waveguides produced from a-Si:H and $\mathrm{SiN}$ can exhibit very low losses, in some cases lower than c-Si, their electronic properties are inferior. On the other hand, poly-Si has the potential to exhibit optical and electronic properties that are comparable with c-Si, but so far its performance has been limited due to small grain sizes that act as scattering and absorption points.

In general, to obtain good quality poly-Si materials they need to be deposited at temperatures higher than $900^{\circ} \mathrm{C}$ $[3,4]$. However, such high temperatures are incompatible with many CMOS processes, resulting in a drive to develop lower temperature methods $\left(<450^{\circ} \mathrm{C}\right)$ [2], though to date the optical losses have remained a limiting factor $[5,6]$. In this work, we report the fabrication of poly-Si waveguides via local laser annealing of a-Si photonic wires. Our technique allows for the fabrication of poly-Si devices with a thermal budget of $320^{\circ} \mathrm{C}$, and is thus suitable for back-end integration with electronics. The crystallization of the processed material has been assessed through microRaman spectroscopy and XRD measurements, confirming the quasi-single crystal characteristics of the photonic wires. Transmission measurements reveal a waveguide loss of $5.13 \mathrm{~dB} / \mathrm{cm}$, which is the lowest loss reported for a low temperature poly-Si material to date.

\section{Starting material and experimental setup}

Our starting sample consists of a $400 \mathrm{~nm}$ film of a-Si that was deposited on a $4.7 \mu \mathrm{m}$ thick silica box layer using a hot-wire chemical vapor deposition technique, at a temperature of $320^{\circ} \mathrm{C}$. The a-Si wire structures were then patterned to have widths of $1.5 \mu \mathrm{m}$ via e-beam lithography and dry etching, as shown in Fig. 1 a). The set up used to anneal the wires is shown in Fig. $1 \mathrm{~b}$ ), where the laser is an Argon ion source delivering $1 \mathrm{~W}$ of continuous wave $(\mathrm{CW})$ radiation at $488 \mathrm{~nm}$. The power on the sample was adjusted using a half wave plate and polarization beam splitter cube. The beam was then focused on the sample using a 10x microscope objective (MO), to achieve a $4.7 \mu \mathrm{m}$ diameter spot. The surface of the sample was imaged on a CCD camera using white light illumination and the position controlled via a set of micro-precision stages.

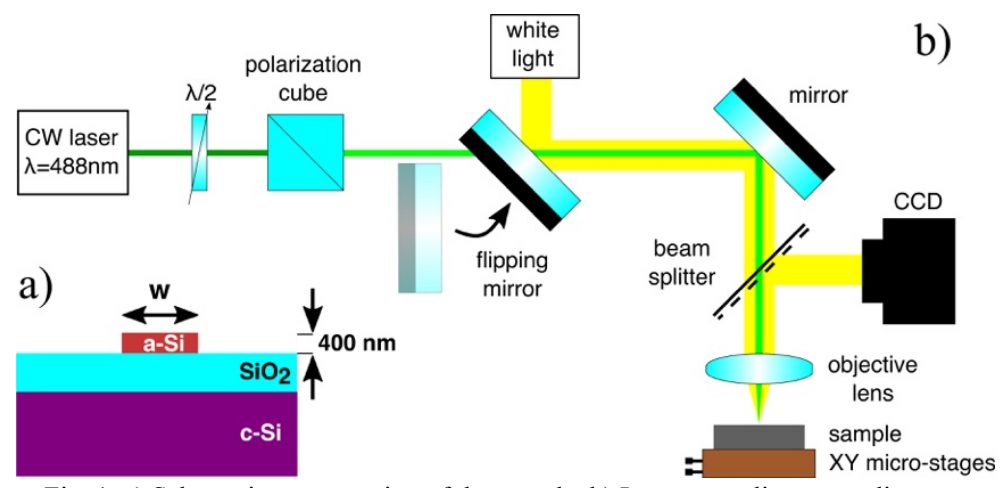

Fig. 1. a) Schematic cross-section of the sample. b) Laser annealing setup diagram. 


\section{Results}

The a-Si wires were crystallized with a laser power of $230 \mathrm{~mW}$, corresponding to an intensity of $1.33 \mathrm{MW} / \mathrm{cm}^{2}$ on the sample surface, and the beam was scanned along the structure at a speed of $0.1 \mathrm{~mm} / \mathrm{s}$. For this combination of laser power and scan speed, sufficient thermal energy was transferred to the wires to completely melt the amorphous material, allowing for the formation of large crystal grains that extend across the entire waveguide width [7]. To verify this, the crystal quality of the annealed material was assessed via micro-Raman spectroscopy. The measured Raman spectra of the waveguide before (blue) and after (red) laser annealing are shown in Fig. 2 a). The deposited material displays a broad spectrum centered at $480 \mathrm{~cm}^{-1}$, typical of a-Si, while the annealed material exhibits a peak centered at $518 \mathrm{~cm}^{-1}$ with a full width at half maximum (FWHM) of $2.74 \mathrm{~cm}^{-1}$, in excellent agreement with the single crystal reference $\left(520 \mathrm{~cm}^{-1}\right.$ and $\left.2.7 \mathrm{~cm}^{-1} \mathrm{FWHM}\right)$ [8]. Further confirmation of the single-crystal like nature was provided by XRD measurements taken using a high energy, micro-focused beam line generated by a synchrotron light source. These studies revealed a single diffraction spot over the entire waveguide width, for lengths $>40 \mu \mathrm{m}$.

To determine the optical quality of our poly-Si waveguides, transmission measurements were performed to establish the optical losses. A $1550 \mathrm{~nm} \mathrm{CW}$ laser diode was coupled into the fundamental mode of the waveguides (TE polarization) using a $63 \mathrm{x}$ MO. The output was then collected using a $25 \mathrm{x}$ MO, focused onto a power meter or CCD camera. The losses were then determined using a cut-back method, where $\sim 1 \mathrm{~mm}$ was polished off the sample length at each step, as shown in Fig. 2 b). The waveguides display linear losses of $5.13 \mathrm{~dB} / \mathrm{cm}$, which is the lowest losses reported for laser annealed poly-Si materials [5], and also lower than the best PECVD poly-Si waveguides with these dimensions [3].

Finally, scanning electron microscopy (SEM) was used to image the as-deposited and laser crystallized poly-Si wires to observe the effects of the crystallization, as shown in Fig. 2 c) and d). It is clear that during the processing, the molten a-Si reshapes due to surface tension, resulting in a crystallized wire with an ultra-smooth top surface to reduce scattering at this interface.
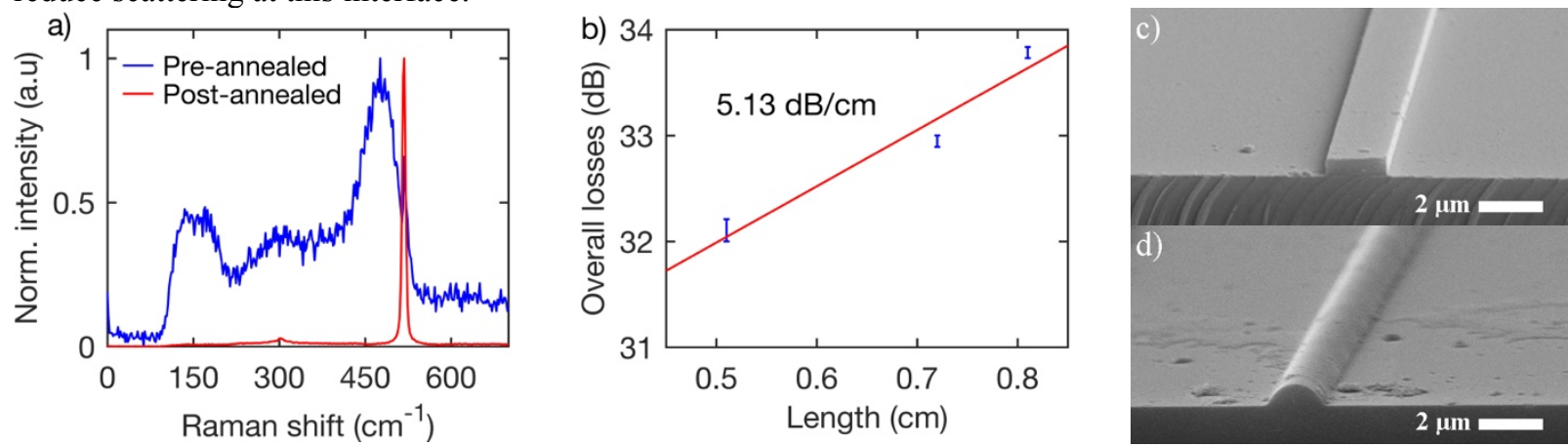

Fig. 2. a) Raman spectra. b) Cut-back loss measurement. c and d) SEM micrograph of a polished waveguide end-facet before and after laser

\section{Conclusions}

We have reported the fabrication of low loss poly-Si waveguides with a thermal budget of $320^{\circ} \mathrm{C}$. Investigations of the crystallographic properties reveal the quasi-single crystal-like nature of the poly-Si material, which is further supported by the low optical losses measured in the waveguides.

Acknowledgment: We thank the Diamond Light Source for access to the I18 beamline and EPSRC for funding.

\section{References}

[1] A. Harke, M. Krause, and J. Mueller, "Low-loss singlemode amorphous silicon waveguides,” Electron. Lett. 41, 1377-1379 (2005).

[2] Y. H. D. Lee and M. Lipson, “Back-End Deposited Silicon Photonics for Monolithic Integration on CMOS,” IEEE J. Sel. Top. Quant. Electron. 19, 8200207 (2013).

[3] J. S. Orcutt, S. D. Tang, S. Kramer, K. Mehta, H. Li, V. Stojanovic, and R. J. Ram, “Low-loss poly-Silicon waveguides fabricated in an emulated high-volume electronic process," Opt. Express 20, 7243-7254 (2012).

[4] Q. Fang, J. F. Song, S. H. Tao, M. B. Yu, G. Q. Lo, and D. L. Kwong, "Low loss ( 6.45dB/cm) sub-micron polycrystalline silicon waveguide integrated with efficient SiON waveguide coupler," Opt. Express 16, 6425-6432 (2008).

[5] Y. H. D. Lee, M. O. Thompson, and M. Lipson, "Deposited low temperature silicon GHz modulator," Opt. Express 21, 26688-26692 (2013).

[6] T. M. Ben Masaud, A. Tarazona, E. Jaberansary, X. Chen, G. T. Reed, G. Z. Mashanovich, and H. M. H. Chong "Hot-wire poly-Silicon waveguides with low deposition temperature," Opt. Lett. 38, 4030-4032 (2013).

[7] N. Healy, S. Mailis, N. M. Bulgakova, P. J. A. Sazio, T. D. Day, J. R. Sparks, H. Y. Cheng, J. V. Badding, and A. C. Peacock, “Extreme electronic bandgap modification in laser-crystallized silicon optical fibres" Nat. Materials 13, 1122-1127 (2014).

[8] A. T. Voutsas, M. K. Hatalis, J. Boyce, and A. Chiang, "Raman spectroscopy of amorphous and microcrystalline silicon films deposited by low-pressure chemical vapor deposition,” J. Appl. Phys. 78, 6999 (1995). 